\title{
FREQÜÊNCIA DE MALFORMAÇÕES CONGÊNITAS DAS EXTREMIDADES EM RECÉM-NASCIDOS
}

\author{
FREQUENCYOFCONGENITALMALFORMATIONS OFTHEEXTREMITIES INNEWBORNS \\ Ricardo Jorge da Silva Pereira ${ }^{*}$ \\ Luiz Carlos de Abrew** \\ Vitor Engracia Valenti*** \\ Waleska Duarte Melo Albuquerque ***** $^{*}$ \\ Sheila Cavalcante Pereira ${ }^{+}$ \\ Ricardo Araújo ${ }^{++}$ \\ Marcos Luiz Ferreira Neto ${ }^{+++}$ \\ Saulo Monteiro dos Santos ${ }^{++++}$
}

Pereira RJdaS, Abreu LCde, Valenti VE, Albuquerque WDM, Pereira SC, Araújo R, Ferreira Neto ML, Santos SMdos. Freqüência de Malformações Congênitas das Extremidades em RecémNascidos. Rev Bras Crescimento Desenvol Hum. 2008; 18(2): 155-162.

Resumo: As malformações congênitas constituem alterações de estrutura, função ou metabolismo presentes ao nascer, que resultam em anomalias físicas ou mentais, podendo ou não ser simples ou múltiplas e de maior ou menor importância clínica. O objetivo é de analisar a freqüência de malformações congênitas das extremidades em maternidades da cidade de Maceió. Realizou-se um estudo de coorte transversal prospectivo em 2500 recém-nascidos no período de julho de 2002 a junho de 2003, junto às maternidades Casa Maternal Santa Mônica, Hospital Memorial Arthur Ramos e Santa Casa de Misericórdia de Maceió. Foram excluídos os recém-nascidos em alto risco e casos de aborto. Cerca de 2,88\% dos recém-nascidos estudados apresentaram algum tipo de anomalia do aparelho locomotor. A anomalia maior mais freqüente foi o pé torto eqüinovaro congênito e a anomalia menor de maior incidência foi o pé calcâneo valgo.

Palavras-chave: Anomalias congênitas; recém-nascido; morbidade; malformações.

\section{INTRODUÇÃO}

As malformações congênitas constituem alterações de estrutura, função ou metabolismo presentes ao nascer, que resultam em anomalias físicas ou mentais, podendo ou não ser simples ou múltiplas e de maior ou menor importância clínica ${ }^{1}$.

Constituem um problema para seus portadores e familiares, acarretando, em muitas oca-

Professor Adjunto. Departamento de Cirurgia da Universidade Estadual de Ciências da Saúde de Alagoas. Programa de pós-graduação em Cirurgia da Universidade Federal de Pernambuco - UFPe. Departamento de Cirurgia da Faculda de de Medicina da Universidade Estadual de Ciências da Saúde de Alagoas - UNCISAL.E-mail: ricardojsp@ig.com.br

** Pós-Doutor em Saúde Pública. Faculdade de Saúde Pública. Universidade de São Paulo - São Paulo. Departamento de Fisiologia. Faculdade de Medicina do ABC. Fisioterapeuta do Centro de Especialidades Clínicas da Prefeitura Municipal de São Bernardo do Campo, SP.

*** Departamento de Medicina, Disciplina de Cardiologia, Universidade Federal de São Paulo. Departamento de Fisiologia da Faculdade de Medicina do ABC.

**** Enfermeira. Mestrado em Terapia intensiva no Instituto Brasileira de Terapia Intensiva - IBRATI

+ Acadêmica. Curso de Medicina da Universidade Federal de Alagoas - UFAL

++ Ortopedista do Instituto de Traumatologia e Ortopedia do Rio Grande do Norte

+++ Professor Adjunto. Universidade Federal de Uberlândia - UFU.

${ }^{++++}$Professor Adjunto. Universidade Federal de Pernambuco - UFPe. 
siões, conseqüências de ordem econômica, psíquica e social. Um recém-nascido com anomalia congênita de um membro representa um desafio para seus pais e para a equipe de profissionais responsáveis pelo tratamento ${ }^{1-4}$.

A etiologia dessas malformações não está bem estabelecida, porém, são muitos os fatores causais. Alguns são genéticos e outros atuam durante o desenvolvimento intra-uterino, existindo também a combinação de diversos fatores ${ }^{5}$.

O espectro das malformações congênitas inclui uma variedade de defeitos que vão desde as mais simples até a ausência total do membro, sendo classificadas conforme o estágio do desenvolvimento na qual ocorreu a alteração, o processo que a provocou ou o resultado final. Essas alterações podem aparecer de forma isolada ou associadas ${ }^{6}$.

As malformações dos membros ocupam a terceira posição em freqüência $(1,07 \%)$ observada nos nascimentos na França ${ }^{7}$, podendo ser consideradas como maiores (aquelas que apresentam conseqüências médias ou cirúrgicas) ou menores (aquelas que apresentam um efeito mínimo sobre a função ou aceitabilidade social). As menores, incluindo as posturais, podem influir na prevalência, porém, em termos práticos, apresentam bom prognóstico e, na maioria das vezes, dispensam qualquer tipo de tratamento ${ }^{8}$.

O exame clínico do recém-nascido é importante e se constitui no principal meio para diagnóstico, exceto em relação à displasia do desenvolvimento do quadril. Os achados clínicos muitas vezes não permitem diagnosticá-la de forma correta e definitiva, de modo que os quadris suspeitos devem ser bem avaliados, através de exame ultrassonográfico. Em muitos países este exame é utilizado rotineiramente em pacientes portadores de alterações clínicas ou que apresentem fatores de risco para a anomalia ${ }^{9-11}$.

Os dados estatísticos brasileiros são controversos em relação à incidência de malformações congênitas, além dos estudos na literatura não considerarem grupos multiétnicos na obtenção dos resultados, o que dificulta a compreensão da incidência aproximada destes distúrbios em diferentes regiões, bem como no país inteiro.
Assim, o objetivo é descrever a prevalência das anomalias das extremidades em maternidades da cidade de Maceió, Estado de Alagoas.

\section{MÉTODO}

\section{População Estudada}

Realizou-se levantamento prospectivo no universo de 2500 recém-nascidos do período de julho de 2002 a junho de 2003, na Casa Maternal Santa Mônica, Hospital Memorial Arthur Ramos e Santa Casa de Misericórdia de Maceió, na cidade de Maceió, Estado de Alagoas. A composição da amostra foi realizada por indivíduos de hospitais público, privado e filantrópico.

As visitas para avaliação dos recém-nascidos foram realizadas nas primeiras 48 horas após o nascimento no alojamento comum e quartos das respectivas maternidades. Foi definido como critério de inclusão único o parto ocorrido numa das três maternidades estudadas. Foram critérios de exclusão: casos de abortos e natimortos e recém-nascidos de alto risco. A pesquisa foi aprovada pelo Comitê de Ética em Pesquisa da Universidade de Ciências da Saúde de Alagoas - UNCISAL.

\section{Coleta de Dados}

O diagnóstico da doença foi estabelecido por equipe multidisciplinar. Buscou-se, clinicamente, identificar deformidades no esqueleto apendicular. Todos os pacientes, com ou sem deformidades, foram submetidos a radiografias, de frente, perfil, bacia e mãos; incidências adicionais foram usadas naquelas que apresentavam deformidades ortopédicas. Utilizou-se uma ficha de avaliação (Anexo 1) para anotação dos achados clínicos da avaliação. Um único pesquisador treinado efetuou todas as avaliações e respectivas anotações. Foi utilizado o exame clínico como critério diagnóstico de malformações, exceto para a displasia de desenvolvimento do quadril, que utilizou o exame de imagem para seu diagnóstico. A análise do exame ultrassonográfico foi realizada por um mesmo avaliador, treinado e especialista em aparelho locomotor, utilizando-se 
sempre o mesmo equipamento de imagem nas três maternidades avaliadas.

\section{RESULTADOS}

Levou-se em consideração a ocorrência da displasia de desenvolvimento do quadril mesmo sendo observado estalido em 20 quadris de recém-nascidos, dado que a avaliação ultrassonográfica se apresentou em aspecto de normalidade. A anomalia maior de maior frequiência encontrada foi o pé torto congênito (tabelas 1, 2 e 3).

$\mathrm{Na}$ Santa Casa de misericórdia foram avaliados 1008 recém-nascidos (456 do sexo feminino), enquanto no Hospital Arthur Ramos 960 recém-nascidos (468 do sexo masculino) foram examinados e na Maternidade Santa Mônica foram analisados 532 recém-nascidos (248 do sexo masculino). A frequiência com relação ao gênero foi similar nos três centros avaliados: 1.268 $(50,7 \%)$ para o gênero masculino e $1.232(49,3 \%)$ para o feminino.
A Tabela 1 descreve o tipo de anomalia segundo sexo em diferentes hospitais. Na Casa Maternal Santa Mônica foi observado uma maior distribuição de recém-nascidos com pé torto congênito, enquanto que a distribuição de polidactilia foi uniforme em todos os locais avaliados, sendo todas do tipo I.

As anomalias maiores, bem como as menores (Tabelas 2 e 3), foram avaliadas nos três hospitais. A ocorrência das anomalias menores e maiores foram semelhantes entre os sujeitos examinados, sendo que o quadro de pé calcâneo congênito foi aquele de maior incidência.

\section{DISCUSSÃO}

Nas deformidades maiores encontradas, apesar de terem sido observados estalidos em 20 quadris de recém-nascidos, não se considerou a ocorrência da displasia de desenvolvimento do quadril, uma vez que os exames do ultrasom da região se apresentaram normais. Assim,

Tabela 1: Freqüência absoluta, freqüência relativa e freqüência acumulada das deformidades maiores diagnosticadas em função do sexo em diferentes locais.

\begin{tabular}{|c|c|c|c|c|c|c|}
\hline Local & Anomalia & Masculino & Feminino & $\begin{array}{l}\text { Freqüência } \\
\text { absoluta \% }\end{array}$ & $\begin{array}{l}\text { Freqüência } \\
\text { relativa } \%\end{array}$ & $\begin{array}{l}\text { Freqüência } \\
\text { acumulada } \%\end{array}$ \\
\hline \multirow{2}{*}{$\begin{array}{l}\text { Casa Maternal } \\
\text { Santa Mônica }\end{array}$} & $\begin{array}{l}\text { Pé torto } \\
\text { congênito }\end{array}$ & 6 & 4 & 10 & 1,87 & 1,87 \\
\hline & Polidactilia & - & 4 & 4 & 0,75 & 2,62 \\
\hline $\begin{array}{l}\text { Hospital Memorial } \\
\text { Arthur Ramos }\end{array}$ & Total & 2 & 2 & 4 & 0,20 & 0,20 \\
\hline \multirow{2}{*}{$\begin{array}{l}\text { Santa Casa de } \\
\text { Misericórdia de Maceió }\end{array}$} & Polidactilia & 4 & - & 8 & 0,79 & 0,79 \\
\hline & $\begin{array}{l}\text { Pé torto } \\
\text { congênito }\end{array}$ & 6 & 2 & 4 & 0,39 & 1,18 \\
\hline
\end{tabular}

Tabela 2: Distribuição das freqüências das anomalias maiores diagnosticadas e freqüência absoluta das anomalias em função do lado afetado em todos os hospitais.

\begin{tabular}{lcc}
\hline Anomalia & $\begin{array}{l}\text { Freqüência } \\
\text { absoluta } \%\end{array}$ & $\begin{array}{l}\text { Freqüência } \\
\text { relativa } \%\end{array}$ \\
\hline Pé torto congênito & 18 & 0,72 \\
Polidactilia & 10 & 0,40 \\
Total & 28 & 1,12 \\
\hline
\end{tabular}

Tabela 3: Distribuição das frequiências das anomalias menores em todos os hospitais.

\begin{tabular}{lcc}
\hline Anomalia & $\begin{array}{c}\text { Freqüência } \\
\text { absoluta } \%\end{array}$ & $\begin{array}{l}\text { Freqüência } \\
\text { relativa \% }\end{array}$ \\
\hline Pé calcâneo valgo & 32 & 1,28 \\
Antepé aducto & 8 & 0,32 \\
Cavalgamento $V$ dedo & 4 & 0,16 \\
Total & 44 & 1,76 \\
\hline
\end{tabular}


as anomalias maiores encontradas foram o pé torto congênito e a polidactilia. O pé torto congênito ocorreu na razão de 1,5 do sexo masculino para cada um do feminino, enquanto a polidactilia somente ocorreu no sexo feminino.

A polidactilia foi a anomalia maior mais freqüente no Hospital Memorial Arthur Ramos, não sendo diagnosticada outras anomalias maiores nos 960 recém-nascidos examinados, sendo observado apenas no sexo feminino.

A incidência para a polidactilia foi de 4 para 1000 nascidos vivos, semelhante à encontrada em João Pessoa por Aguiar ${ }^{10}$. As formas pré e pós-axial ocorreram com freqüências semelhantes. A polidactilia afeta mais comumente os dedos laterais da mão, sendo o polegar e o dedo mínimo os mais comprometidos ${ }^{10}$. Todos os casos encontrados em nosso estudo foram diagnosticados como sendo do tipo I e não houve predomínio de raça.

O diagnóstico da polidactilia é clínico e geralmente feito pelo neonatologista no momento do parto. A inspeção mostra um número maior de dedos em relação ao normal. Muitas vezes se associa a outras deformidades e sua presença torna obrigatória a pesquisa em outros sistemas ${ }^{11}$.

As deformidades menores encontradas ocorreram com uma frequiência semelhante às deformidades maiores. Destaca-se que esta categoria possui bom prognóstico evolutivo, independentemente do tratamento efetuado ${ }^{8}$.

$\mathrm{Na}$ França, as anomalias dos membros superiores e inferiores são as mais freqüentes das más-formações $(1,07 \%)$ observadas nos nascimentos ${ }^{7}$. Os obstáculos para determinação da prevalência são grandes e este fato se deve à dificuldade de acesso aos recém-nascidos, principalmente com o estabelecimento de casas maternais em todos os municípios. Nestas casas, a exemplo de muitas maternidades da capital, a gestante é assistida apenas pela parteira e as crianças sequer têm suas funções vitais examinadas.

Em Maceió, não existe trabalho semelhante ao deste estudo para que se possam comparar os resultados obtidos. Farias Filho ${ }^{8}$ reforça a idéia de que os estudos populacionais deveriam ser realizados de forma prospectiva, por siste- mas e por especialistas. Fiddian ${ }^{12}$ e Engesaeter ${ }^{13}$ não são da mesma opinião, visto que defendem a idéia de que esses exames possam ser realizados por fisioterapeutas e neonatologistas sem prejuízo nos resultados.

Neste estudo, previamente foram estabelecidos critérios para diagnóstico das anomalias dos membros inferiores. Pela existência de muitos trabalhos que atestem a ineficácia dos exames clínicos no diagnóstico da displasia de desenvolvimento do quadril, levou-se em conta a presença desta anomalia apenas quando existia alteração no exame ultrassonográfico, considerado seguro para estabelecimento do diagnóstico.

Pela sistematização estabelecida, obtevese uma incidência para as anomalias maiores de 2,62\% na Casa Maternal Santa Mônica, 0,2\% no Hospital Arthur Ramos, 1,18\% na Santa Casa de Misericórdia de Maceió e 1,12\% quando se considerou os três hospitais. Para as anomalias menores, encontrou-se o valor de $1,76 \%$, no total. $\mathrm{O}$ valor encontrado para o Hospital Arthur Ramos fugiu à realidade encontrada nos trabalhos publicados, enquanto que os demais resultados encontram respaldo na literatura pesquisada ${ }^{9,10,14}$.

Estima-se que em torno de 3\% dos recém-nascidos apresente alguma anomalia do aparelho locomotor, valor equivalente ao trabalho realizado em João Pessoa ${ }^{8}$ e que está de acordo com os dados da literatura pesquisada, cujos valores oscilam entre $2 \%$ e $3 \%$.

A anomalia encontrada com maior frequiência neste trabalho foi o pé torto equiinovaro congênito, com uma prevalência de 7,2 para 1000 nascidos vivos. A proporção foi de 1,5 do sexo masculino para um feminino. Além disso, o pé torto congênito também foi a anomalia de maior freqüência considerando-se o número de todos os três hospitais, predominando no sexo masculino. Foram diagnosticados oito pacientes com pé torto unilateral e dez pacientes na forma bilateral. Esta prevalência é maior que a apresentada por Farias Filho ${ }^{8}$ em João Pessoa. No Brasil, a prevalência varia entre 2,17 e 6,84 por 1000 . Uma mesma pesquisa realizada anteriormente em João Pessoa mostrou uma prevalência de 5,4 por 1000.

Infere-se que a prevalência do pé torto 
eqüinovaro congênito seja elevada, o que se pode observar na prática clínica, em que diariamente são atendidos pacientes portadores dessa anomalia $^{8}$. Em 1988, numa avaliação de 2.401 recém-nascidos, foi encontrado a deformidade em 100 crianças $(4,2 \%) .{ }^{15}$ Estes achados sustentam outro estudo ${ }^{16}$, o qual afirmou ser esta a deformidade congênita mais comum do pé. Em nosso estudo, em relação ao gênero, a proporção foi de dois do sexo masculino para um do sexo feminino. Em 1988 foi afirmado que comprometimento unilateral é o mais freqüente ${ }^{17}$, porém, segundo a literatura, a deformidade é bilateral em cerca de $50 \%$ dos $\operatorname{casos}^{18}$. Em nosso estudo, foram diagnosticados oito pacientes com pé torto unilateral e dez pacientes na forma bilateral, o que é compatível com a literatura. Apesar de o pé torto congênito estar relacionado a antecedentes genéticos, não observamos o comprometimento de familiares dos portadores das anomalias $^{17}$.

Em nosso estudo, todos os casos encontrados de pé torto congênito estiveram associados a comprometimentos ortopédicos (pé torto idiopático). O diagnóstico do pé torto congênito idiopático é freqüentemente feito ao nascimento, a partir da observação clínica da deformidade. Outras causas dessa condição podem ser anormalidades do tecido de conexão (problemas do colágeno e outras proteínas causando fibrose intensa e rigidez), alterações neurológicas (alterações da medula espinhal, ex: mielomeningocele - defeito de formação; ou paralisia cerebral defeito no sistema nervoso central que causa desequilíbrios musculares, entre outros) ${ }^{19}$. Durante os anos 60, os resultados dos tratamentos do pé torto congênito eram analisados separadamente, os aspectos clínicos e os radiológicos ${ }^{19}$, havendo discordância entre estes. Historicamente, as avaliações radiográficas do pé torto congênito foram consideradas por diversos autores ${ }^{20}$, fato que sustenta nosso método de avaliação.

Em relação à displasia de desenvolvimen- to do quadril, embora existam relatos de 1:1000 nascidos vivos em relação à displasia de desenvolvimento do quadril ${ }^{21}$, neste estudo (Tabelas 1 e 2) não foi diagnosticada esta anomalia pelos critérios previamente estabelecidos. Os estalidos muitas vezes podem estar associados a movimentos de fáscias ou frouxidão cápsulo-ligamentar. Em 1960 foi relatado que existe uma maior concentração de estrogênio no sangue dos recém-nascidos com displasia, determinando assim uma maior frouxidão capsular nos quadris destas crianças $^{21}$. Em 1994, foi destacado que os exames de Ortolani e de Barlow são variáveis conforme o examinador ${ }^{21-23}$.

O que se discute na atualidade não é mais a importância da ultrassonografia, já comprovada e sim sua acurácia, no sentido de que haja uma melhor definição dos critérios para diagnóstico, como bem relata Roposch et $\mathrm{al}^{24}$ (2007) onde avalia imperfeições existentes em muitos trabalhos publicados sobre ultrassonografia do quadril para diagnóstico de displasia de desenvolvimento do quadril.

As séries publicadas na literatura ${ }^{25,26}$ mostram que o diagnóstico de displasia do quadril nem sempre pode ser estabelecido no momento do nascimento ${ }^{23}$. Existe um consenso atual de que o exame clínico não traz segurança e que a ultra-sonografia deve ser utilizada, inclusive porque este exame pode mostrar alterações em quadris considerados clinicamente normais.

Em conclusão, de acordo com as maternidades estudadas, 2,88\% dos recém-nascidos apresentam algum tipo de anomalia do aparelho locomotor das extremidades. A anomalia maior mais freqüiente foi o pé torto eqüinovaro congênito e a anomalia menor de maior incidência foi o pé calcâneo valgo.

Reitera-se que as anomalias menores são de bom prognóstico e que as anomalias maiores necessitam de acompanhamento ambulatorial e de procedimentos intervencionistas para restabelecer sua função. 


\begin{abstract}
Congenital malformations are structural, functional or metabolic alterations present in birth that cause physical or cognitive anomalies; they may be simple or multiple and of major or minor clinical importance. We aimed to analyze the frequency of locomotor apparatus congenital anomalies (extremities) in maternities of the city of Maceió. A prospective cross-sectional cohort study was carried out with 2500 newborns between July 2002 and June 2003 in the maternities Casa Maternal Santa Mônica, Hospital Memorial Arthur Ramos and Santa Casa de Misericórdia de Maceió. Approximately $2.88 \%$ of the studied newborns presented some type of locomotor apparatus anomaly. The most frequent major anomaly was the equinovarus clubfoot and the most frequent minor anomaly was the valgus calcaneus foot. It is important to note that an early diagnosis improves the quality of life of this population.
\end{abstract}

Keywords: Abnormalities; newborn; morbidity; malformations.

\section{REFERÊNCIAS}

1. Fembach SA. Common orthopedic problems of the newborn in Nurse. Clin North Am. 1998; 33: 583-594.

2. Mason K.J. Congenital orthopedic anomalies and their impact on the family in Nurse. Clin North Am. 1991; 26: 1-16.

3. Garcia MG, Ferreira EAP, Oliveira FPS. Análise da compreensão de pais acerca do Teste do Pezinho. Rev bras crescimento desenvolv hum. 2007: 17(1); 1-12.

4. Abreu LC, Souza AMB, Oliveira AG. Incidência de hemorragia peri-intraventricular em recémnascidos pré-termo e a relação com o peso ao nascer. Rev bras crescimento desenvolv hum. 2007: 17(2); 24-30.

5. Penaloza C, Orlanski S, Ye Y, Entezari-Zaher T, Javdan M, Zakeri Z. Cell death in mammalian development. Curr Pharm Des. 2008; 14(2): 18496.

6. Chen CP. Chromosomal abnormalities associated with neural tube defects (II): partial aneuploidy. Taiwan J Obstet Gynecol. 2007; 46(4):336-51.

7. Taillemite JL. Malformations congènitales des membres: embryologie, étiologie in Encycl. Méd. Chir. Paris Appareil locomoteur. 1975; 15A: $10-12$.

8. Farias Filho OC. Malformações congênitas do aparelho locomotor na cidade de João Pessoa: prevalência e aspectos prognósticos. [dissertação]. Recife: Universidade Federal de Pernambuco; 1997.

9. Brandt C. Malformações Congênitas. Papel na morbimortalidade infantil. JBM. 1986; 50: 101114.

10. Aguiar RCT, Figueredo Neto JA, Dahia PL, Cisneiros GLF, Nunesmaia HGS. Malformações congênitas em João Pessoa, Paraíba. C C S. 1986; 8: 31-38.

11. Carvalho Júnior AE, Fernandes TD, Corsato MA, Masagão RA, Freitas MF, Barbosa AP, Salomão O. Indicações de técnicas cirúrgicas no tratamento da polidactilia do pé. Rev Bras Ortop. 1999; 33(7): 537-543.

12. Fiddian NJ, Gardiner JC. Screening for congenital dislocation of the hip by Physiotherapists. Result of ten-year study. J Bone Joint Surg. 1994; 76(B) (3): 458-459.

13. Engesaeter LB, Wilson DJ, Nag D, Benson MKD. Ultrasound and congenital dislocation of the hip. J Bone Joint Surg. 1990; 72(B) (2): 197201.

14. Leite JCL. Anomalias do membro superior: uma perspectiva genético-clínica em Clinica Ortopédica. 2003; 4(1): 25-30.

15. Jones DA, Powell N. Ultrasound and neonatal hip screening. A prospective study of "high risk" babies. J Bone Joint Surg. 1990; 72(B) (3): 457-459.

16. Pereira RJS. O pé torto congênito. In: Jornada Norte-Nordeste de Cirurgia Pediátrica. Maceió, 1987.

17. Riordan DC, Bayne LG. La extremidad superior. In: Ortopedia Pediátrica $2^{\mathrm{a}}$ ed. 689-692 Panamericana Buenos Aires, 1988, p. 689-92

18. Malik S, Grzeschik KH. Synpolydactyly: clinical and molecular advances. Clin Genet. 2008; 73(2): 113-20.

19. Attenborough CG. Severe congenital talipes equinovarus. J Bone Joint Surg Br. 1966;48(1):319.

20. Shazly EM, Trainor B, Kernohan WG, Turner I, Haugh PE, Johnston AF et al. Reliability of the Barlow and Ortolani tests for neonatal hip instability. J Med Screen. 1994; 1: 165-168.

21. MacEwen GD, Bunnell WP, Ramsey PL. La 
Cadera. In: Lovell WW, Winter RB. Ortopedia Pediátrica $2^{a}$ ed. 705-713 Panamericana Buenos Aires, 1988, p. 705-13.

22. Terjesen BT, Berg V. Ultrasound for hip assessment in the newborn. J Bone Joint Surg. 1989; 71(B) (5): 767-773.

23. Clarke NMP, Clegg J, Al-Chalabi AN. Ultrasound screening of hip at risk for CDH. J Bone Joint Surg. 1989; 71(B) (1): 9-12

24. Roposch A, Moreau NM, Uleryk E, Doria AS. Developmental dysplasia of the hip: quality of reporting of diagnostic accuracy for US. Radiol 2006;241(3):854-60.

25. Bar-On E, Meyer S, Harari G, Porat S.

Ultrasonography of the hip in developmental hip dysplasia. J Bone Joint Surg. 1998; 80(B) (2): 321-324.

26. Milani C, Laredo Filho J, Ishida A., Ascêncio JB. Nakagawa JRM. A ultrassonografia do quadril do recém-nascido pelo método de Graf. Rev Bras Ortop. 1993; 28(1-2): 25-32. 
Quadro 1: Questionário.

$\mathrm{N}^{\mathrm{o}}$ Nome:

Idade GS

Gesta Para Endereço

Pré-natal: Parto: normal cesariana indicação

Idade gestacional: Peso RN APGAR Sexo

Cor: Apresentação Estatura PC PT

Deformidades observadas: anotar na figura abaixo

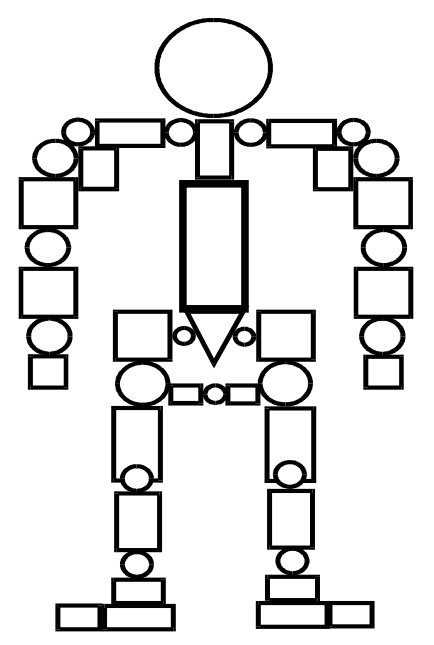

$\square$ Lesão

$\nabla$ Região Não Examinada

图 Com Lesão (descrever lesão)

Torcicolo - Pseudoartrose congênita da clavícula - Paralisia obstétrica Sinostose rádio-ulnar - Deformidades na mão e dedos -- Escoliose Luxação congênita do quadril e joelhos - Deformidades nos pés e dedos (pé torto, astragalo vertical etc) - Encurtamentos - Processos infecciosos (lues congênita - artrite) - Processos traumáticos - fraturas outros

Observações:

Lesões Encontradas + Exames Solicitados

Dados Sobre o RN - Deformidades associadas + hereditariedade etc. 